\title{
Multi-sensor Formation Targets Template Matching Tracking Algorithm
}

\author{
Haipeng Wang, Shuyi Jia* and Ziling Wang \\ Institute of Information Fusion, Naval Aeronautical and Astronautical University, Yantai, China \\ ${ }^{*}$ Corresponding author
}

\begin{abstract}
Aiming to solve the track refined tracking problem of the formation targets with the multi-sensor detections, based on the relative invariant of the actual positions of the formation targets in each detection period, a new algorithm named multisensor formation targets template matching tracking algorithm was proposed. In the algorithm, the template shape matrix and the shape matrix to be matched were respectively obtained with the previous associated formation state set and formation measurement set. The least-cost matching matrix was obtained with the matching search model and the matching matrix validation rules. Moreover, based on the template and the corresponding matching matrix, the state update of each track within the formation targets was completed with the Kalman filter. The analysis results of the simulation data show that obvious advantages of this algorithm are established in the aspects of tracking accuracy, real-time performance and effective tracking rate, compared with the multi-sensor multiplied hypothesis algorithm based on data compressing technical which is a superior performance algorithm in the traditional multisensor multi-target tracking field. The real engineering requirement of the refined tracking of the formation targets is met very well with this algorithm.
\end{abstract}

Keywords- template matching; multi-sensor; formation targets; refined tracking

\section{INTRODUCTION}

The problem of target tracking using multiple sensors in complex formation in the background with the improvement of sensor resolution becomes a research hotspot in recent years [1 8]. Because the distance is very close between the inner target formation and the movement patterns are similar, the cross-connects, error results are easy produced in clutter.

In this paper, template matching methods is used to measure the difference between real targets and clutter, and multi-sensor formation targets template matching [9 12] tracking (called MSFT-TM) algorithm is proposed. Several simulation environments close to actual tracking background are designed, and the validation and analysis are carried out on the tracking performance of the algorithm in this paper.

\section{SYSTEM DESCRIPTION}

Wherever Times is specified, Times Roman or Times New Roman may be used. If neither is available on your word processor, please use the font closest in appearance to Times. Avoid using bit-mapped fonts if possible. True-Type 1 or Open
Type fonts are preferred. Please embed symbol fonts, as well, for math, etc.

The problem of centralized multi-sensor formation target tracking in clutter achieve each goal status updates using the sensor measuring which are reported to fusion center by NS censors.

In this assumption: Each measure most likely to come from a goal; each target has a real echo at each time at most; some sensors may not provide measurements at each interval.

The dynamic equation of system is set as:

$$
\begin{gathered}
\boldsymbol{X}^{t}(k+1)=\boldsymbol{F}(k) \boldsymbol{X}^{t}(k)+\boldsymbol{\Gamma}(k) \boldsymbol{V}^{t}(k) \\
k=1,2, \cdots ; t=1,2 \cdots T_{g}
\end{gathered}
$$

where, $\boldsymbol{X}^{t}(k+1) \in \boldsymbol{R}^{n}$ is global state vector at $k+1$ time ; $\boldsymbol{F}(k) \in \boldsymbol{R}^{n}$ is State transition matrix; $T_{g}$ is the number of the kth target formations of; $\boldsymbol{\Gamma}(k) \in \boldsymbol{R}^{n, h}$

Is noise distribution matrix; $\boldsymbol{V}^{t}(k)$ is discrete time white noise sequence, and $E\left[\boldsymbol{V}^{t}(k)\right]=0, \quad E\left[\boldsymbol{V}^{t}(k) \boldsymbol{V}^{t}(k)^{\mathrm{T}}\right]=\boldsymbol{Q}^{t}(k)$.

$m_{k_{i}}\left(i=1,2, \ldots, N_{s}\right)$ is set as the measurement number from the sensor $i$ at $k$ time, in multi-sensor environment, the measurement equation is expressed as:

$$
\begin{gathered}
\boldsymbol{Z}_{l}^{i}(k)=\boldsymbol{H}_{i}(k) \boldsymbol{X}^{t}(k)+\boldsymbol{W}_{l}^{i}(k), \\
t=1,2, \ldots, T ; l=1,2, \ldots, m_{k_{i}}
\end{gathered}
$$

where, $\boldsymbol{Z}_{l}^{i}(k)$ is the $l$ th measurement at $k$ time from the $i$ th sensor; $\boldsymbol{H}_{i}(k)$ is the measurement matrix; $\boldsymbol{W}_{l}^{i}(k)$ is zero mean Gaussian noise vector with known covariance and statistically independent, $T$ is the target number; the comprehensive observation vector obtained from the fusion center at $k$ time is:

$$
\boldsymbol{Z}(k)=\left\{\boldsymbol{Z}_{i}^{s}(k)\right\} \quad i=1, \cdots, m_{s} ; s=1, \cdots, N_{s}
$$

And the measurement errors between each sensor are assumed to be statistically independent. 


\section{MSFT-TM ALGORITHM}

\section{A. The Pre-internet based on the Overall Formation}

$\boldsymbol{G}(k-1)$ is set as the updates value set of formation track status and the value of covariance:

$\boldsymbol{G}(k-1)=\left\{\boldsymbol{X}_{n}^{t}(k-1), \boldsymbol{P}_{n}^{t}(k-1)\right\} t=1, \cdots, T_{g}(k-1) ; n=1, \cdots, N_{g}^{t}(k-1)$

Where, $T_{g}(k-1)$ is the formation number at $k-1$ time; $N_{g}^{t}(k-1)$ is the target number of the th formation; For the measurement set of the i-th set of sensors, the formation presplit is made by the cycle threshold method, $\boldsymbol{g}_{l}^{i}(k)=\left\{\boldsymbol{z}_{j}^{i l}(k)\right\}, j=1, \cdots, J_{l}^{i}(k) \quad, \quad J_{l}^{i}(k) \quad$ is the measurement number of $\boldsymbol{g}_{l}^{i}(k)$.

$$
\overline{\boldsymbol{z}}_{l}^{i}(k) \quad \text { is the of the } \boldsymbol{g}_{l}^{i}(k) \text { center, }
$$
$\overline{\boldsymbol{G}}^{t}(k-1)=\left[\overline{\boldsymbol{X}}^{t}(k-1), \overline{\boldsymbol{P}}^{t}(k-1)\right]$ is the center track $\boldsymbol{G}^{t}(k-1)$ updates value of formation track status and the value of covariance. In this case, the formation measurements and formation of interconnected track are temporarily transformed into the interconnection between $\overline{\boldsymbol{z}}_{l}^{i}(k)$ and $\overline{\boldsymbol{G}}^{t}(k-1)$.

Same as the traditional objectives, the step predictive value of $\overline{\boldsymbol{X}}^{t}(k-1)$ is set as the center, associated wave gate is set up. If $\overline{\boldsymbol{z}}_{l}^{i}(k)$ meet the formula (4), the target is considered to fall within the associated wave door of $\overline{\boldsymbol{z}}_{l}^{i}(k)$.

$$
\left[\bar{z}_{l}^{i}(k)-\boldsymbol{H}_{i}(k) \overline{\boldsymbol{X}}^{t}(k \mid k-1)\right]^{\mathrm{T}} \overline{\boldsymbol{S}}_{i l}^{-1}(k)\left[\bar{z}_{l}^{i}(k)-\boldsymbol{H}_{i}(k) \overline{\boldsymbol{X}}^{\mathrm{t}}(k \mid k-1)\right] \leq \gamma
$$

where, $\gamma$ is a constant threshold; $\overline{\boldsymbol{S}}_{i l}(k)$ is the new covariance.

Typically, the distance is far between the formation and the formation, the probability of falling into the same associated door is small; if there are measurements of multiple formations for the same sensor fall into the same associated wave door, the nearest formation measurement is selected for of the amount of the association based on nearest neighbors method. Formation measurement in associated with $\boldsymbol{G}^{t}(k-1)$ of measurement successfully reported by each sensor set is provided as:

$$
\underline{\boldsymbol{g}}^{t}(k)=\left\{\underline{\boldsymbol{g}}_{i}^{t}(k)\right\}, i=1, \cdots, N_{s}^{\prime}
$$

where, $N_{s}^{\prime}$ is the number of sensors for the association formation measurement.

\section{B. Establishing a Template Matching Model}

First, confirm that you have the correct template for your

Based on $\boldsymbol{G}^{t}(k-1)$ and $\boldsymbol{g}^{t}(k)$ pre-associated successfully, the template matching model is established, the clutter is eliminated and the internet is achieved between the track of the formation and the real measurements, the process is divided into the following four steps.

\section{1) Establishing the matrix of template shape}

A template is established based on $\boldsymbol{G}^{t}(k-1)$, the rectangle A is obtained as shown in Fig.2.

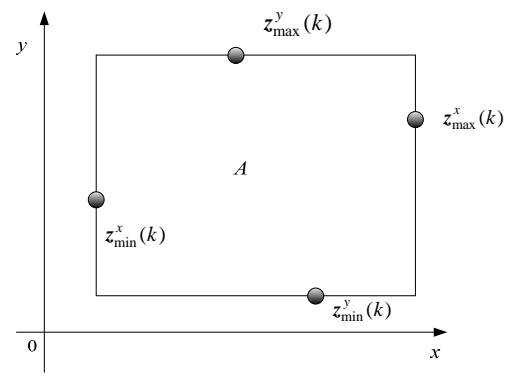

FIGURE I. SCHEMATIC DIAGRAM OF MEASUREMENT AREA

$\boldsymbol{z}_{j}^{i l}(k)$ is expressed as:

$$
\boldsymbol{z}_{j}^{i l}(k)=\left\{\begin{array}{lr}
H\left(\boldsymbol{\omega}_{i}, \boldsymbol{\omega}_{t}\right)+\boldsymbol{w}_{j}^{i l}, & \text { measurements from target } \\
\boldsymbol{a}_{j}^{i l}, & \text { measurements from clutter }
\end{array}\right.
$$

where, $\boldsymbol{w}_{j}^{i l}=\left[w_{j x}^{i l}, w_{j y}^{i l}\right]$ is random noise subject to Gaussian distribution; $\omega_{i}$ is the location of the sensor i at k time; $\omega_{t}$ is the true location of the target $\mathrm{t} ; \boldsymbol{a}_{j}^{i l}$ is subjected to uniform distribution within the sensor detection area. When no maneuvering happen, the graphics composed of target's position within the fleet at temporary moment is basically the same; the measurements value are moved affected by measurement error. By the formula (6), the swing range of is $2 w_{j x}^{i l}, 2 w_{j y}^{i l}$ approximately in $x, y$ direction. A rectangle is divided into a some small rectangles by way of Figure $7, \mu_{x}$, $\mu_{y}$ are classified foundation in $\mathrm{x}, y$ direction which are defined as

$$
\left\{\begin{array}{l}
\mu_{x}^{\mathrm{m}}=\min \left\{4 \max \left\{w_{j x}^{i l}\right\}, x_{\min }^{c}(k-1)\right\} \\
\mu_{y}^{\mathrm{m}}=\min \left\{4 \max \left\{w_{j y}^{i l}\right\}, y_{\min }^{c}(k-1)\right\}
\end{array}\right.
$$

where, $x_{\min }^{c}(k-1), y_{\min }^{c}(k-1)$ are the minimum difference of each target $\boldsymbol{G}^{t}(k-1)$ in $x, y$ direction. 


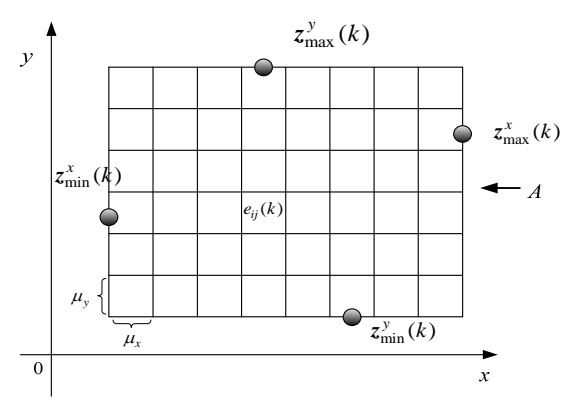

FIGURE II. SEGMENTATION OF RECTANGLE A

The matrix of template shape of rectangle $\mathrm{A}$ is defined as:

$$
\boldsymbol{E}_{\mathrm{m}}^{t}(k-1)=\left\{e_{i j}^{\mathrm{m}}\right\} i=1, \cdots, l_{x}^{\mathrm{m}} ; j=1, \cdots, l_{y}^{\mathrm{m}}
$$

where, $l_{x}^{\mathrm{m}}, l_{y}^{\mathrm{m}}$ are the number of rectangle A inx, $y$ direction;

$$
e_{i j}=\left\{\begin{array}{c}
1, \text { existing measurement in the corresponding small matrix } \\
0, \text { no measurement in the corresponding small matrix }
\end{array}\right.
$$

\section{2) Establishing the shape of the matrix to matched}

$\underline{\boldsymbol{g}}^{t}(k)$ is set as the object to be matched, the establishment of a shape to be matched matrix description of specific divided into the following three steps:

a) Based, to find $x$, the maximum and minimum $y$ directions, to build a rectangular $B$.

b) According to the rectangle $B$ is divided into a plurality of small rectangles, and which is as defined in formula (7).

c) Based on the measured fall has immeasurable small rectangle to be established to match the shape of the matrix.

$$
\boldsymbol{E}_{\mathrm{P}}^{t}(k)=\left\{e_{i j}^{\mathrm{P}}\right\} i=1, \cdots, l_{x}^{\mathrm{P}} ; i=1, \cdots, l_{y}^{\mathrm{P}}
$$

where, $e_{i j}^{\mathrm{P}}$ is the same as defined in formula (9). The measurement set $\hat{Z}_{i j}^{t}$ in a small rectangle $e_{i j}^{\mathrm{P}}$ is recorded as:

$$
\widehat{Z}_{i j}^{t}=\left\{\widehat{\boldsymbol{z}}_{l}(k)\right\} \quad l=1, \ldots, \hat{m}_{i j}^{\mathrm{p}}
$$

where, $\hat{m}_{i j}^{\mathrm{p}}$ is the measurement number of $\underline{\boldsymbol{g}}_{i}^{t}(k)$ falling into the $e_{i j}^{\mathrm{P}}$.

\section{3) Matching searching model}

As shown in Fig.2, of the minimum rectangle of rectangular $\mathrm{B}$ in $x, y$ direction is set as a starting point, rectangle $\mathrm{A}$ is embedded in the rectangle $\mathrm{B}$, based on
$\boldsymbol{E}_{\mathrm{m}}^{t}(k-1)$ and $\boldsymbol{E}_{\mathrm{P}}^{t}(k)$ the matching matrix of $\mathrm{B}_{i j}$ and $\mathrm{A}$ is established as:

$$
\boldsymbol{P}^{t}=\left[p_{i j}\right], i=1, \ldots, m_{x}^{\prime \mathrm{p}} ; j=1, \ldots, m_{y}^{\prime \mathrm{p}}
$$

where, $m_{x}^{\prime \mathrm{p}}, m_{y}^{\prime \mathrm{p}}$ is the matching number in $x, y$ direction, and

$$
\left\{\begin{array}{l}
m_{x}^{\mathrm{p}}=l_{x}^{\mathrm{P}}-l_{x}^{\mathrm{m}}+1 \\
m_{y}^{\prime \mathrm{p}}=l_{y}^{\mathrm{P}}-l_{y}^{\mathrm{m}}+1
\end{array}\right.
$$

$$
p_{i j}=\sum_{i=1}^{l_{x}^{\mathrm{m}} \cdot l_{y}^{\mathrm{m}}} p_{i^{\prime} j^{\prime}}^{\mathrm{m}} \quad i^{\prime}=1, \ldots, l_{x}^{\mathrm{m}} ; j^{\prime}=1, \ldots l_{y}^{\mathrm{m}}
$$

where,

$$
P_{i^{\prime} j^{\prime}}^{\mathrm{m}}= \begin{cases}1 & \text { if } e_{i^{\prime} j^{\prime}}^{\mathrm{m}}=e_{\left[(i-1) l_{x}^{\mathrm{m}}+i^{\prime}\right]\left[(j-1) l_{y}^{\mathrm{m}}+j^{\prime}\right]}^{\mathrm{p}} \\ 0 & \text { else }\end{cases}
$$

If $p_{i j}=l_{x}^{\mathrm{m}} l_{y}^{\mathrm{m}}$, it is assumed that $\mathrm{A}$ matches $\mathrm{B}_{i j}$, that the internal structure of each measurement are similar between $\mathrm{A}$ and $\mathrm{B}_{i j}$.

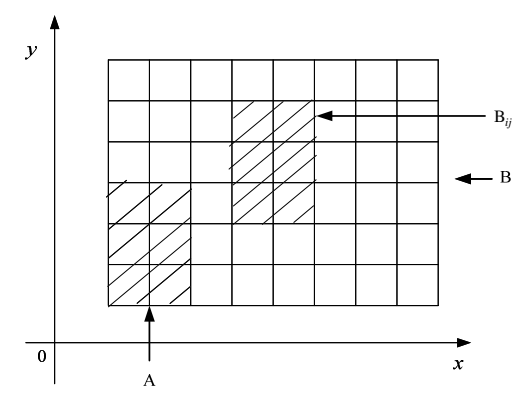

FIGURE III. MATCHING SEARCH MODEL

\section{4) Matching matrix}

There are several $B_{i j}$ which satisfies the matching condition in rectangle $\mathrm{B}$, in this condition the final match matrix is determined by building match cost matrix. based on Match matrix $P_{s}^{t}$ of sensor s, the definition for the match cost matrix $\boldsymbol{L}_{s}^{t}$ is:

$$
\boldsymbol{L}_{s}^{t}=\left\{\varsigma_{i j}^{s}\right\} \quad i=1, \ldots, m_{x}^{\prime \mathrm{p}} ; j=1, \ldots, m_{y}^{\prime \mathrm{p}}
$$




$$
S_{i j}^{s}= \begin{cases}\sum_{i^{\prime}=1}^{l_{x}^{\mathrm{m}}} \sum_{j^{\prime}=1}^{l_{y}^{\mathrm{m}}} d_{i^{\prime} j^{\prime}} & p_{i j}=l_{x}^{\mathrm{m}} l_{y}^{\mathrm{m}} \\ 0 & p_{i j} \neq l_{x}^{\mathrm{m}} l_{y}^{\mathrm{m}}\end{cases}
$$

where, $d_{i^{\prime} j^{\prime}}$ is defined as:

$$
d_{i j^{\prime}}= \begin{cases}\min _{l=1: \hat{m}_{i j}^{\mathrm{p}}}\left[\bar{z}_{l}(k)-\hat{z}^{t}(k \mid k-1)\right] & e_{i^{\prime} j^{\prime}}^{\mathrm{m}}=e_{\left.\left[(i-1) l_{x}^{\mathrm{m}}+i^{\prime}\right][(j-1))_{y}^{\mathrm{m}}+j^{\prime}\right]}^{\mathrm{p}}=1 \\ 0 & e_{i j^{\prime}}^{\mathrm{m}}=e_{\left[(i-1) l_{x}^{\mathrm{m}}+i^{\prime}\right]\left[(j-1) l_{y}^{\mathrm{m}}+j^{\prime}\right]}^{\mathrm{p}}=0\end{cases}
$$

At the same time, $\tilde{\boldsymbol{Z}}_{i j}=\left\{\tilde{\boldsymbol{z}}_{i^{\prime} j^{\prime}}\right\}$ is saved in $\mathrm{B}_{i j} \cdot \boldsymbol{L}_{s}^{t}$ is established for s sensors, $\mathrm{B}_{i j}^{*}$ is match matrix, interconnection measurement set of each grid in $\mathrm{B}_{i j}^{*}$ is $\tilde{Z}_{i j}^{*}$.

\section{Track Status Updates within the Formation}

And based on $\boldsymbol{G}^{t}(k-1)$ and $\tilde{\boldsymbol{Z}}_{i j}^{*}$, the templates A and matching rectangle $\mathrm{B}_{i j}^{*}$ are used, if $\boldsymbol{X}_{n}^{t}(k-1)$ fall into the small rectangle of $\mathrm{A}$, based on the measurement $\tilde{\boldsymbol{z}}_{i^{\prime} j^{\prime}}^{*}$ falling into $\mathrm{B}_{i j}^{*}$, the status and covariance updates of $\boldsymbol{X}_{n}^{t}(k-1)$ are completed using kalman filtering.

\section{SimUlation}

To verify the performance and effectiveness of the algorithm, two typical target formation movement are set, the performance of MSFT-TM algorithm is analyzed from three aspects of tracking algorithm accuracy, timeliness, effective track rate of the tracking, and compared with Centralized multisensor multipled hypothesis algorithm based on data compressing Technic ${ }^{[13]}$ (called Data Compression CMSMHT) algorithm based on data compression among the traditional multi-sensor multi-target tracking algorithms with superior performance.

\section{A. Simulation Environment}

Define abbreviations and acronyms the first time they are used in the text, even after they have been defined in the abstract. Abbreviations such as ASME, SI, MKS, CGS, sc, dc, and rms do not have to be defined. Do not use abbreviations in the title or heads unless they are unavoidable.

Three 2D radar sensors are assumed, in order to compare the performance of tracking algorithms in different simulation environment, the following two typical environment are set.

Environment 1: Simulation of two intersecting tight formation objectives of the campaign. Eight goals are provided in the presence of a two-dimensional plane, and constitute two formations which are made uniform linear motion. The first four goals compose of the first formation, the initial position of each object is $(5000 \mathrm{~m}, 800 \mathrm{~m}),(5200 \mathrm{~m}, 1000 \mathrm{~m}),(5550 \mathrm{~m}$,
$1200 \mathrm{~m}),(5700 \mathrm{~m}, 1400 \mathrm{~m})$, respectively, are the initial velocity $(-200 \mathrm{~m} / \mathrm{s}, 300 \mathrm{~m} / \mathrm{s}) ; 5$ to 8 target composition consisting of the first two formations, the initial position of each object, respectively $(-5000 \mathrm{~m}, 8000 \mathrm{~m}),(-5200 \mathrm{~m}, 8200 \mathrm{~m}),(-5550 \mathrm{~m}$, $8400 \mathrm{~m}),(-5700 \mathrm{~m}, 8600 \mathrm{~m})$, both the initial velocity $(100 \mathrm{~m} / \mathrm{s}$, $300 \mathrm{~m} / \mathrm{s})$.

Environment 2: To verify the time-consuming and effectively track changes in rate with clutter of each algorithm on the basis of Environment 1, clutter values are shown in Table 1 as below.

TABLE I. THE NUMBER OF CLUTTER IN ENVIRONMENT

\begin{tabular}{|c|c|c|c|c|c|c|}
\hline$\lambda_{1} /$ unit & 1 & 2 & 3 & 4 & 5 & 6 \\
\hline$\lambda_{2} /$ unit & 2 & 4 & 6 & 8 & 10 & 12 \\
\hline
\end{tabular}

B. Simulation Results

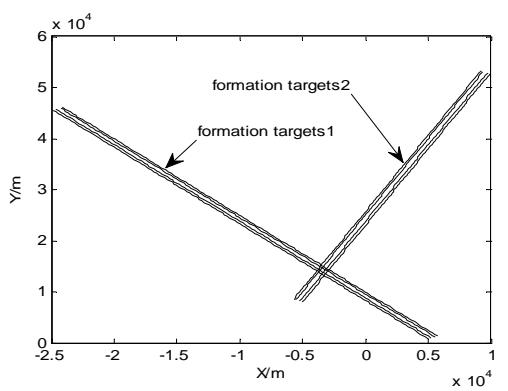

FIGURE IV. TRUE STATE OF THE FORMATION TARGETS

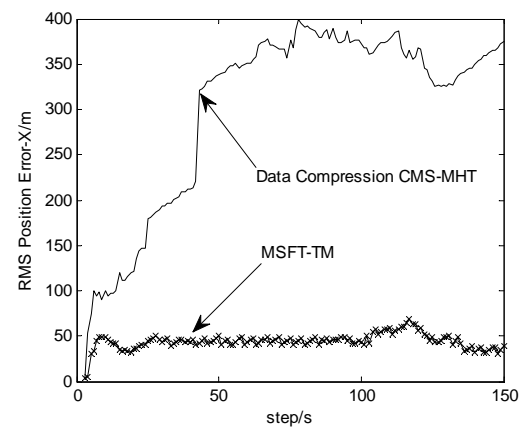

FIGURE V. RMS POSITION ERROR AT X-AXIS

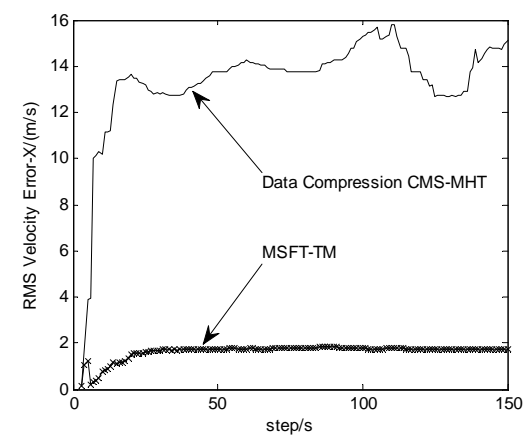

FIGURE VI. RMS VELOCITY ERROR AT X-AXIS 


\section{Simulation Conclusion}

Figure 4 is a target formation's environment situation in Environment 1, it can be seen from the figure that two formations are cross movement. Figures 5 and 6 , in Environment 1, CMSRT-GT-TM algorithms and data compression CMS-MHT algorithm, respectively are the comparison chart of RMS position and speed difference; it can be seen from the figure, the algorithm can effectively track the target, RMS position error is less than $60 \mathrm{~m}$, rms velocity error is less than $2 \mathrm{~m} \mathrm{/} \mathrm{s}$; and based on the data compression algorithm CMS-MHT to track the effect is not ideal, after step
50 the RMS position error is up to $300 \mathrm{~m}$ or more, the root mean square speed error is up to $12 \mathrm{~m} / \mathrm{s}$ or more, in high precision tracking actual occasions has been unable to meet the engineering requirements.

Traditional multi-sensor multi-target tracking algorithm and the existing tracking algorithms are difficult to meet the engineering needs for formation tracking. Several simulation environments close to actual tracking background are designed, and the validation and analysis are carried out on the tracking performance of the algorithm in this paper.

TABLE II. EFFECTIVE TRACKING RATIO AND RUNTIME OF THE ALGORITHMS VARIED WITH THE NUMBER OF CLUTTER

\begin{tabular}{|c|c|c|c|c|c|c|c|}
\hline \multirow{2}{*}{ Clutter number } & $\lambda_{1}$ /unit & 1 & 2 & 3 & 4 & 5 & 6 \\
\cline { 2 - 8 } & $\lambda_{2} /$ unit & 2 & 4 & 6 & 8 & 10 & 12 \\
\hline \multirow{2}{*}{$\begin{array}{c}\text { Effective } \\
\text { tracking rate }\end{array}$} & MSFT-TM & 1 & 0.9875 & 0.9523 & 0.9034 & 0.8923 & 0.7534 \\
\cline { 2 - 8 } & Data Compression CMS-MHT & 0.8569 & 0.7746 & 0.6512 & 0.6058 & 0.5860 & 0.3934 \\
\hline $\begin{array}{c}\text { time } \\
\text { consuming }\end{array}$ & MSFT-TM & 0.0234 & 0.0783 & 0.1534 & 0.1946 & 0.2578 & 0.3208 \\
\cline { 2 - 8 }$y$ & Data Compression CMS-MHT & 0.1480 & 0.1956 & 0.2525 & 0.3678 & 0.6502 & 0.9946 \\
\hline
\end{tabular}

Table 2 for the environment 2 both algorithms effectively track rate and Algorithm takes the clutter with a few changes in the comparison table.

As can be seen from the table 2, corresponding to the same number of clutter, effective tracking rate CMSRT-GT-TM data compression algorithm is significantly higher than the CMS-MHT algorithm, when the number of clutter $(6,12)$, the effective rate of the former track above $75 \%$, while the latter was only $39.34 \%$ effective tracking, can not meet the actual needs of the project. In addition, with the increase in the number of clutter, two algorithms effectively track rate of decline, data compression algorithm CMS-MHT declines significantly greater than the algorithm.

As shown from Table 3, with the increasing in the number of clutter, a single update takes two algorithms were all increased; data compression rate of increase large CMS-MHT algorithm, when the number of clutter $(6,12)$, its single update has reached 0.9946 millisecond time-consuming, in real-time requirements higher real occasions can not meet the engineering requirements; clutter corresponding to the same number, CMSRT-GT-TM algorithm takes significantly smaller single update.

\section{CONCLUSION}

Traditional multi-sensor multi-target tracking algorithm and the existing tracking algorithms are difficult to meet the engineering needs for formation tracking.

Several simulation environments close to actual tracking background are designed, and the validation and analysis are carried out on the tracking performance of MSFT-TM algorithm in this paper.

\section{ACKNOWLEDGMENT}

This work is supported by National Nature Science Foundations of China (Grant No. 61531020 and 61471383)

\section{REFERENCES}

[1] Geng Wendong. Summarizing of group-target tracking[C]. The 10th China Radar Conference, 2008: 367-371.

[2] Xing Fengyong, Xiong Wei, Wang Haipeng. A formation target track initiation algorithm based on clustering and hough transform[J]. Journal of Naval Aeronautical and Astronautical University, 2010, 25(6): 624629

[3] Peng Z H, Sun L, Chen J. Path Planning of Multiple UAVs Low-altitude Penetration Based on Improved Multi-agent Coevolutionary Algorithm[C]//2011 30th Chinese Control Conference, 2011, 7: 40564061.

[4] Zhen Q, Shelton C R. Improving Multi-target Tracking via Social Grouping[C]//2012 IEEE Conference on Computer Vision and Pattern Recognition, 2012, 7: 1972-1978.

[5] Zhao Mengmeng, Zhang Qun, Chen Yijun. A Sliding Window Tracking Algorithm for Distingushing Space Group Targets[J]. Journal of Austronautics. 2015, 36(10): 1187-1194.

[6] Lyudmila M. Group Object Structure and State Estimation in The Presence of Measurement Origin Uncertainty[C]. 2009 IEEE 15th Workshop on Statistical Signal Processing, 2009, 8: 473-476.

[7] Feldmann M, Franken D. Advances on Tracking of Extended Objects and Group Targets Using Random Matrices[C]. 12th International Conference on Information Fusion, 2009, 7: 1029-1036.

[8] Lian F, Han C Z, Liu W F. Sequential Monte Carlo Implementation and State Extraction of the Group Probability Hypothsis Density Filter for Partly Unresolvable Group Targets-tracking Problem[J]. IET Radar, Sonar and Navigation, 2010, 4(5): 685-702.

[9] Abdul R M, Mahalekshmi M C. Automated Object Recognition and Pattern Matching Analysis of Runways Using Surface Track Data[C]. 2011 3rd International Conference on Electronics Computer Technology, 2011, 4: 46-51. 
[10] Masood-Ur-Rehman M, Fang J C, Saffih F. Automatic Star Pattern Recognition in Tracking Mode[C]. 2009 6th International Bhurban Conference on Applied Sciences and Technology, 2009, 1: 249-252.

[11] Graciano A B V, Cesar R M, Bloch I. Graph-based Object Tracking Using Structural Pattern Recognition[C]. 2007 Brazilian Symposium on Computer Graphics and Image Processing, 2007, 10: 179-186.

[12] Badri J, Tilmant C, Lavest J M. Hybrid Sensors Calibration: Application to Pattern Recognition and Tracking[C]. 2007 IEEE International Symposium on Intelligent Signal Processing, 2007, 10: 1-5.

[13] Zhang Jingwei, Xiong Wei, He You. Multisensor Multipled Hypothesis Algorithm based on Data Compressing Technic[J]. Journal of Beijing University of Aeronautics and Astronautics, 2007, 33(12):1448-1451. 